\title{
Mechanism of non-thermal effect of Millimeter Wave irradiation on Cell Growth
}

\author{
Ayan Barbora ${ }^{1,3}$, Shailendra Rajput ${ }^{2}$, Konstantin Komoshvili ${ }^{3}$, Jacob Levitan ${ }^{3}$, Asher \\ Yahalom $^{2,4}$ and Stella Liberman-Arono ${ }^{1}$ \\ ${ }^{1}$ Department of Molecular Biology, Ariel University, Ariel, Israel \\ ${ }^{2}$ Department of Electrical \& Electronic Engineering, Ariel University, Ariel, Israel \\ ${ }^{3}$ Department of Physics, Ariel University, Ariel, Israel \\ ${ }^{4}$ Princeton University, Princeton, New Jersey 08543, USA \\ * Corresponding author: Stella Liberman Aronov, Department of Molecular Biology, Ariel \\ University, Kiryat Hamada, POB 3, Ariel, Israel
}

Tel: (972) 3-937-1431

Fax: (972) 3-937-1422

E-mail: Stellar@ariel.ac.il

Conflict of interest: none

Grant sponsor: Ariel Center for Applied Cancer Research

Running title: The effects of $85-105 \mathrm{GHz}$ MMWs on yeast growth 


\begin{abstract}
Nonionizing millimeter-waves (MMW) are reported to inhibit cell division of lung cancer cells. In this article, we present a mechanism for the effect of inhibited cell division upon 85-105 GHz MMW irradiation. Strains of cell division model organism Saccharomyces cerevisiae cultured under physiological conditions were analyzed for the effects of MMW exposure. Irradiated cells showed a reduced growth rate than that of control (sham) cells. DNA damage repair mutant (rad52) strain cells were also subjected to MMW exposure to identify the involvement of genomic alteration(s) in this process. Irradiated wild type and rad52 mutant strains showed similar colony growth profiles indicating MMW treatment does not alter genomic DNA. Further, MMW interaction with cytological water was explored as a possible mechanism of action. Cells absorbed more power as compared to plain water. MMW irradiation highly absorbed by the cytological water content likely affects proteomic changes, accounting for the observed effects of inhibited cell division. Irradiations using a standard horn antenna were compared to that of a compact waveguide for increased power which led to complete termination of cell division. Our results provide indications of the development of non-invasive nonionizing irradiation procedures to treat tumor metastasis and control microbial infections.
\end{abstract}

Keywords: Biomedical applications, millimeter wave, non-invasive devices, yeast 


\section{Millimeter Waves in Biological Irradiation}

The influence of millimeter wave (MMW) radiation on biological systems is a topic of considerable importance because of two important reasons: 1) to establish safety standards for the use of MMWs for communications, 2) to understand the mechanisms of interaction between MMW and living systems. These investigations opened the door to new potential applications of MMW in the field of biomedical engineering including selective targeting of cancer cells. MMW in the range $75-110 \mathrm{GHz}$ (so called W-band) are classed as nonionizing radiation because of the low energy of their photons in the range of 0.3-0.4 meV.

In medical sciences, cancer is considered one of the deadliest diseases for humans and is very difficult to diagnose at early stages [1]. Cancer is known to arise from accumulated mutations in oncogenes leading to uncontrolled tumor cell growth [2,3]. Currently used radiation therapy in cancer treatment gives rise to many detrimental side effects [2] including the development of other more dangerous cancers due to ionizing radiation (involved in such treatments) resulting in mutagenesis [3]. In very recent work, we showed that the MMW irradiations $(75-105 \mathrm{GHz})$ with a non-thermal power density of $0.2 \mathrm{~mW} / \mathrm{cm}^{2}$ caused morphological changes in H1299 human lung cancer cells [4] leading to targeted mortality [5]. MMWs are also reported to be helpful to detect different types of cancers [6]. The MMW technologies are also applicable in the treatment of several diseases, like gastrointestinal disorders, wound healing, remote monitoring of wounds, non-invasive detection of glucose levels, pain relief, diabetes, dermatitis, etc. [7, 8]. However, the mechanism of the therapeutic application on pathological specimens is not well understood and is one of the main obstacles to the wide-scale use of this technology.

Saccharomyces cerevisiae yeast cells are frequently used as a model system for in-vitro studies, as yeast is the simplest eukaryotic organism with a nucleus. Many essential cellular processes in yeast and humans are the same, which makes yeast suitable to study basic molecular processes transferrable to similar biological process in humans. Characteristics of tumor cell growth are studied using models of yeast cell division $[3,9,10]$. Among the lower eukaryotic organisms, yeast is evolutionarily closer to higher eukaryotic mammalian cells than either bacteria or plants [11]. Humans are multicellular organisms, and their inherent cell biology is dependent on the cytoplasm containing proteins, carbohydrates, and lipids. About $23 \%$ of the yeast genome is conserved with human cells, including all the corresponding biological functions and biochemical pathways remaining the same [11]. In the laboratory, yeast cells are cheap and simple to grow, culture, and experiment. Earlier studies on irradiation of aqueous suspensions of wild type Saccharomyces cerevisiae yeast culture are highly ambiguous. These studies reported either no change or increased/decreased rate of growth upon microwave irradiation of $42 \mathrm{GHz}$ and $50 \mathrm{~mW}$ power $[12,13]$. The authors reported the exclusion of thermal effects in such procedures by continuously monitoring temperature during the duration of exposure. On the other hand, MMW irradiation of yeast cells in the range of $41.650-41.798 \mathrm{GHz}$ for $4 \mathrm{~h}$ and $20 \mathrm{~mW}$ power found frequency sensitive results with increased cell growth at some frequencies and reduced at other values [14]. Another study confirmed the increased growth rate of yeast upon irradiation with $968 \mathrm{MHz}$ for $7 \mathrm{~h}$ at $17 \mathrm{dBm}$ power [15]. Results of such studies on the interaction of millimeter waves with biological samples are often met with inconsistence and non-reproducibility, as they do not rely on characterizing biological functions like change in genetic material or protein structure to correlate with the observed 
effects [16]. Knowledge of the mechanism of action is needed to gain trust in the use of MMW technology for clinical applications.

In this article we explore the effect of MMW (85-105 GHz) irradiation on Saccharomyces cerevisiae yeast as a model of eukaryotic cell division. The MMW are propagated using a standard pyramidal horn antenna. The radiated power density and power distribution across the antenna aperture are analyzed. The influence of MMW irradiation on yeast cells was manifested in the retarded cell growth effect. Irradiation of rad52 mutant cells showed that reduction in the cell growth was not due to genetic DNA damage Using a waveguide delivering higher energy achieved complete termination of cell division. Further, this study suggests possible mechanisms of retarded cell growth due to MMW exposure encouraging further biomedical applications in clinical settings and research work.

\section{Conditions of Cell Culture}

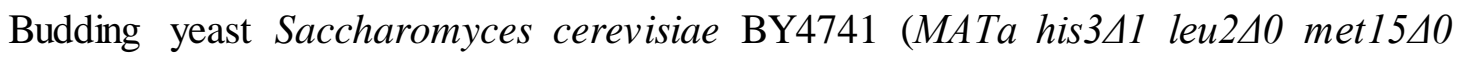
ura3 $\Delta 0$ ) wild type (WT) strain and its rad52 mutant strain BY4741 (MATa his3 1 leu2 $\Delta 0$

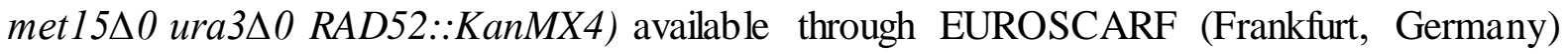
were used for the irradiation. Cells were grown in standard synthetic complete (SC) liquid medium at a temperature of $30^{\circ} \mathrm{C}$. The growth rate of both control and irradiated cells were measured using an absorbance plot at $600 \mathrm{~nm}$ measured by a standard spectrophotometer in units of optical density (OD). Cultures were adequately diluted to 0.1 OD using a standard absorbance plot at the start of experiment and incubated until they reached an OD value of 0.4 (the point at which cells initiate the logarithmic growth phase). Cultures at 0.4 OD were diluted to $10000,1000,100$ and 50 cells/ $\mu$ l (to determine the optimal concentration of cells and energy dosage). 1-2 $\mu$ l volume of those solutions were dropped onto SC agar plates. Six colonies were seeded in two replicates: one for irradiation and another for comparison as control (sham). After irradiation, the cells were transferred to SC liquid medium and incubated under standard conditions. Growth rate of both irradiated and control (sham) yeast cells were measured regularly at intervals of 90 minute over a period of $\sim 8 \mathrm{~h}$ to assay the effect of MMW exposure on physiological growth. 


\section{Conditions of Irradiation}

The schematic diagram of the experimental setup for MMW irradiation is illustrated in Fig. 1. For experiments, MMWs $(85-110 \mathrm{GHz})$ were generated using the signal generator (Keysight technology, N5183B, $9 \mathrm{kHz}-20 \mathrm{GHz}$ ), and $\times 6$ active frequency multiplier (Quinstar Tech Inc., QMM-311220025). A standard gain pyramidal horn antenna (Quinstar Tech Inc., QWH-WPRROO) was used for MMW emission. The power of transmitted waves from the horn was measured using identical horn antenna and digital storage oscilloscope (Agilent technology, DSO-X 2004A). The well-known Friis transmission formula was employed for the calculation of transmitted power in the far-field region. Distribution of relative energy across antenna aperture was measured using open ended waveguide (Quinstar Tech Inc., QWHWPRROO) in the near field (Fig. 2). A detailed description of the irradiation setup was also described in our previous work [17].
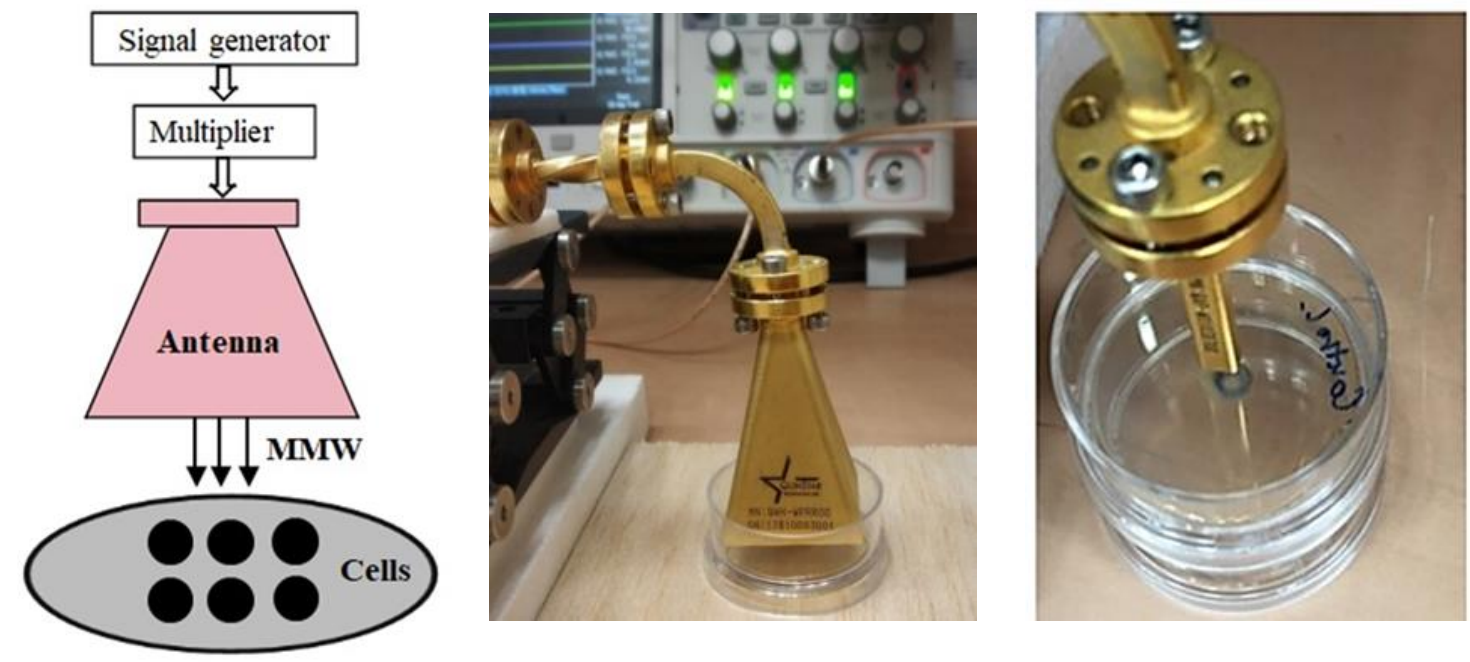

Fig. 1. Block diagram of the experimental setup for irradiation and picture of the pyramidal antenna and wave guide.

The yeast cells were spotted on the Agar medium for irradiation. Cells were exposed to specific frequencies of $85 \mathrm{GHz}, 95 \mathrm{GHz}$, and $105 \mathrm{GHz}$ at $5 \mathrm{dBm}$ power over 6 hours respectively for each under a continuous irradiation regime. The irradiation experiment for each frequency was repeated six times. In order to scrutinize the effect of power and time of irradiation, cells were exposed at a constant frequency of $105 \mathrm{GHz}$ MMW for different durations (5 and $6 \mathrm{~h}$ ) and power densities $(5 \mathrm{dBm}$ and $6 \mathrm{dBm})$. The power levels of 5 and 6 $\mathrm{dBm}$ correspond to 3.16 and $3.98 \mathrm{~mW}$ absolute powers, respectively. The average power density at the aperture of the horn antenna is shown in Table 1.

Table 1. Average energy flux at the aperture of the antenna for different frequencies.

\begin{tabular}{|c|c|c|}
\hline $\begin{array}{c}\text { Frequency } \\
(\mathrm{GHz})\end{array}$ & $\begin{array}{c}\text { Power density } \\
\left(\mathrm{mW} / \mathrm{cm}^{2}\right)\end{array}$ & $\begin{array}{c}\text { Time of } \\
\text { irradiation }(\mathrm{h})\end{array}$ \\
\hline 85 & $1.39 \pm 0.03$ & 6 \\
\hline 95 & $1.04 \pm 0.02$ & 6 \\
\hline 105 & $0.83 \pm 0.02$ & 5 \\
\hline 105 & $0.83 \pm 0.02$ & 5 \\
\hline
\end{tabular}




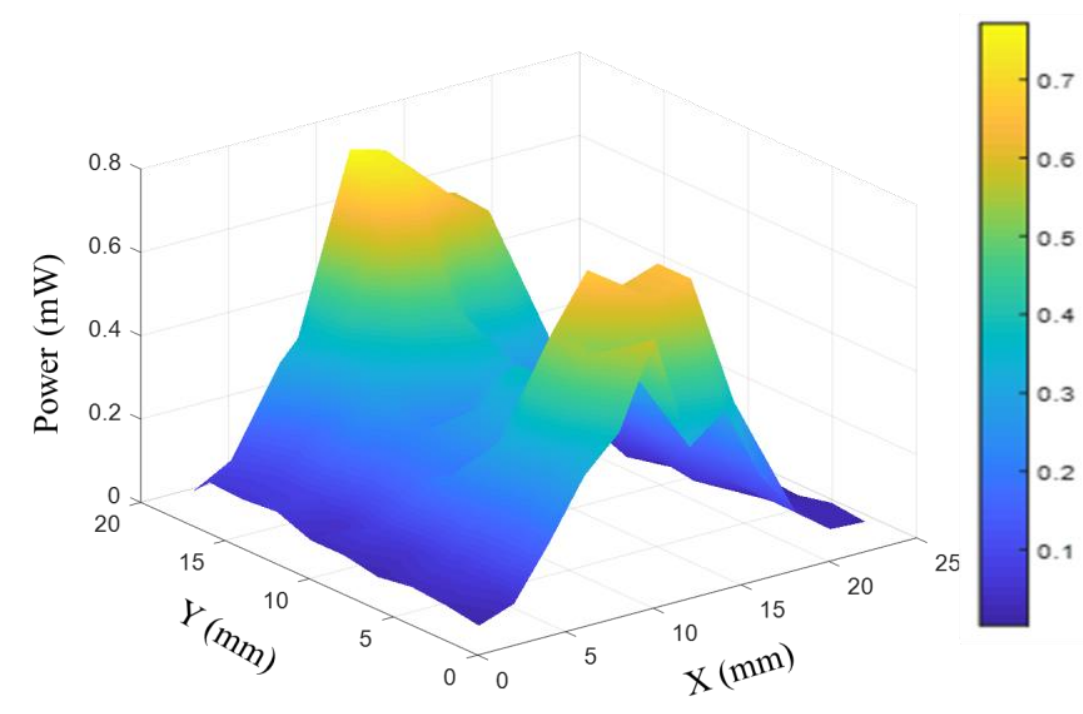

Fig. 2. Relative distribution of power across antenna aperture.

\section{MMW irradiation reduces the rate of cell division in a frequency dependent manner}

In our previous experiment, haploid WT cells (BY4741 strain) did not demonstrate an effect with respect to their growth rate and cell viability after 5-6 hours irradiation with MMW of $75 \mathrm{GHz}$ frequency [17]. Here, we demonstrate (Figure $3 \mathrm{a}$ and $3 \mathrm{~b}$ ) the growth rate results of both WT control (sham) and irradiated cells at frequencies above $75 \mathrm{GHz}(85,95,105)$. Both control and irradiated cells were subjected to the same culture conditions. The six distinct irradiated yeast colonies were exposed at the same time. Subsequent to the irradiation treatment; the growth rate and division of yeast cells were examined by incubating them under standard conditions. It is observed that the MMW irradiation at all examined frequencies affect the growth rate of WT yeast strain and reduces the rate of division up to $62 \%$ as compared to sham (control) (Fig. 3a). Delay in cell proliferation becomes significantly noticeable over 3-5 hours of physiological incubation post-irradiation treatment. Effect of different duration of irradiation ( $5 \mathrm{~h}$ versus $6 \mathrm{~h}$ ) and power densities $(5 \mathrm{dBm}$ versus $6 \mathrm{dBm})$ at a constant frequency of $105 \mathrm{GHz}$ (delivering the same energy dosage) was examined subsequently.
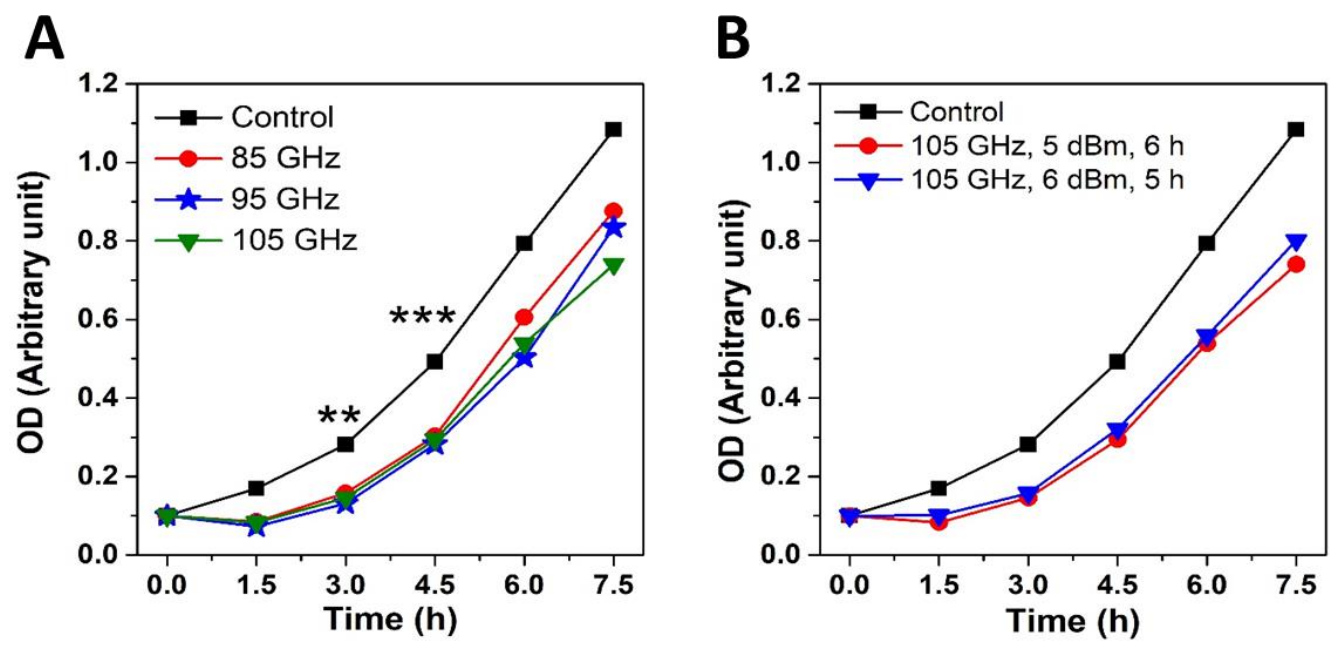
Fig. 3. (a) Growth of BY4741 Saccharomyces cerevisiae (50 cells/ $\mu$ l) cells measured in OD units after irradiation for 6 hours at $5 \mathrm{dBm}$. Plots indicate mean values of growth rate of six separate yeast colonies exposed together at the same time $(\mathrm{n}=6)$ for each frequency; Single Factor Anova analysis $(* *$ indicates p-value $<0.01$ and $* * *$ indicates $\mathrm{p}$-value less than <0.001) (b) Growth of irradiated sample for different time durations and powers at constant frequency of $105 \mathrm{GHz}$ (and constant dose energy).

Figure $3 \mathrm{~b}$ demonstrates that the inhibited cell division effect is MMW frequency and energy dependent. Single frequency at $105 \mathrm{GHz}$ was used as it affects WT yeast cell growth to the same extent as for all the other examined MMW frequencies (ref. Fig 3a). The agar layer thickness is kept constant throughout.

\section{MMW wave irradiation does not alter genetic DNA}

The effects of irradiation were observed to be persistent in the irradiated cells even after termination of exposure across six separate experiments. These effects do not arise from thermal effects, as has been reported earlier [18]. Studies in the field of MMW therapy deems irradiation under $1 \mathrm{~mW} / \mathrm{cm}^{2}$ as not to give rise to thermal effects in living cells [14]. And since our experiments involved a power density of about $1 \mathrm{~mW} / \mathrm{cm}^{2}$ placing the results in a nonthermal range, it is necessary to investigate other possible mechanism(s) responsible for the decreased growth rate of MMW irradiated cells. Therefore, the next step of the investigation is to check for the presence of genomic alteration in the irradiated cells. In this direction, we examined for genetic change in DNA using the rad52 mutant strain. RAD52 is a protein required to repair DNA double-strand breaks. This protein is absent in the rad52 mutant strains, and such yeast cells die upon irradiation by DNA damaging electromagnetic spectra [19, 20]. Both WT and mutant types of cells were exposed to $90 \mathrm{GHz}$ MMW at $5 \mathrm{~dB}$ power for $6 \mathrm{~h}$ and subsequently incubated for colony growth. It was observed that both types of cells showed similar colony growth profiles, demonstrating that reduced cell growth is not due to genetic DNA damage (Fig. 4).

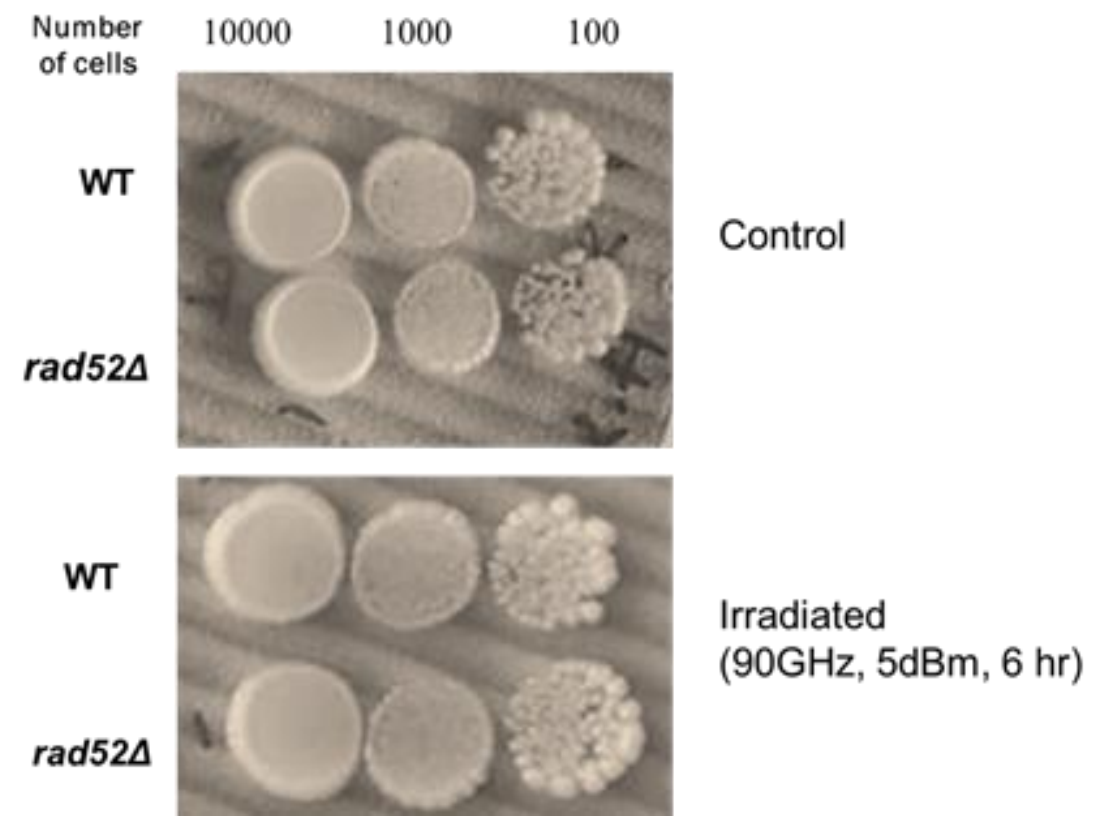


Fig. 4. Colony growth profile of WT and rad52 mutant cells subjected to MMW irradiation and incubated at $30^{\circ} \mathrm{C}$.

In general, non-ionizing radiations are not expected to alter DNA. Edwards et al. proposed a mechanism of coherent frequency-specific deposition of microwave energy on DNA in aqueous solution [21]. The resonance of DNA molecules with irradiated spectra can be calculated in terms of the absorption coefficient. 2734 base pairs (bp) supercoiled circular DNA, 2734 bp linear DNA, 1786 bp linear DNA, and 948 bp linear DNA was found to resonate with $2.55-8.75 \mathrm{GHz}, 2.75-5.60 \mathrm{GHz}, 4.10 \mathrm{GHz}$, and $2.65 \mathrm{GHz}$ respectively [21]. The polymer chain length determining the structural conformation and size (globular or linear, large or small) are hence directly correlated to the resonant frequency. Illustratively, resonance shift occurs in the frequency range of $41-52 \mathrm{GHz}$ upon changing the length of the haploid genetic material in E. coli [22]. Further, relative viscosity measurements showed that the resonance frequencies decreased proportionally to the enhancement of haploid genome length. Such resonance interactions occur energetically without causing genetic alteration. Illustratively, irradiation of HCE-T and SRA01/04 cell lines by $60 \mathrm{GHz}$ at $1 \mathrm{~mW} / \mathrm{cm}^{2}$ found no statistically significant genotoxic effects on the nucleus [23].

\section{MMW interaction with water as a factor for reduced cell growth}

In the above section, we concluded that the MMW irradiation causes other nonpermanent genetic changes within the cells, which results in their reduced cell growth/division. It is understood in cell biology that the genome encodes the genetic information for hereditary purposes. Leaving aside the genome, the living characteristics of cells are manifested by the interplay of the proteins. Water is a significant constituent of the cell cytoplasm, the site of all biochemical reactions which give rise to biological functions of growth, division, and genetic inheritance in living organisms. Water is also known to absorb electromagnetic radiation in the microwave and infrared spectrum [24]. Studies have looked into and reported resonance absorption of different ranges of wavelengths of this spectrum on biochemical and biological samples.

A biological cell can be supposed to be a compartmentalized structure separated from the surrounding environment by the cell membrane. It has been reported that $65 \mathrm{GHz}$ irradiation reduced the effects of heliogeophysical factors on yeast cells due to the destabilization of intracellular water structure [25]. Under physiological conditions, yeast cells are reported to have $65 \%$ water by composition [26]. Biological functions at the cellular level are affected by proteins, and the functionality of proteins is, in turn, determined by their molecular structure. Proteins are polypeptide chains composed of sequentially joined amino acids folding into the lowest energy conformations in their physiological environment to give rise to threedimensional structures. These structures are essential for the protein's biochemical interactions with other molecules which give rise to biological functions. Changes in the aqueous environment translate into changing the properties of biomolecules.

The results suggest that the proteome is likely to be affected by the interaction of water with MMW irradiation. This accounts for the observed phenomenon of cell growth inhibition without genetic perturbation. Structurally, water is a physical participant during the collapse of the polypeptide chain in protein folding through hydrophobic collapse [27]. Thus, water interacts with proteins to affect their dynamics. Conversely, changes in the chemical composition of the aqueous environment can alter the three-dimensional structure of proteins. 
Molecular transfer model (MTM) predicts conformational changes in protein structures when $\mathrm{pH}$ changes occur in a solution using calculated partition functions of polypeptides [28]. Illustratively, Nitrophorin 4 (NP4) is a protein that releases nitric oxide (NO) in a pH-sensitive manner. NP4 remains in a closed conformation and tightly binds $\mathrm{NO}$ at $\mathrm{pH} 5.5$ [29]. At $\mathrm{pH} 7.5$, deprotonation occurs, changing the conformation and releasing NO.

Figure 5 presents absorbed power over time as a ratio of incident and reflected powers for cells spotted on SCagar, plain water and blank SC agar respectively. The irradiation conditions were kept constant at $85 \mathrm{GHz}(5 \mathrm{dBm})$ for stringency of analysis.

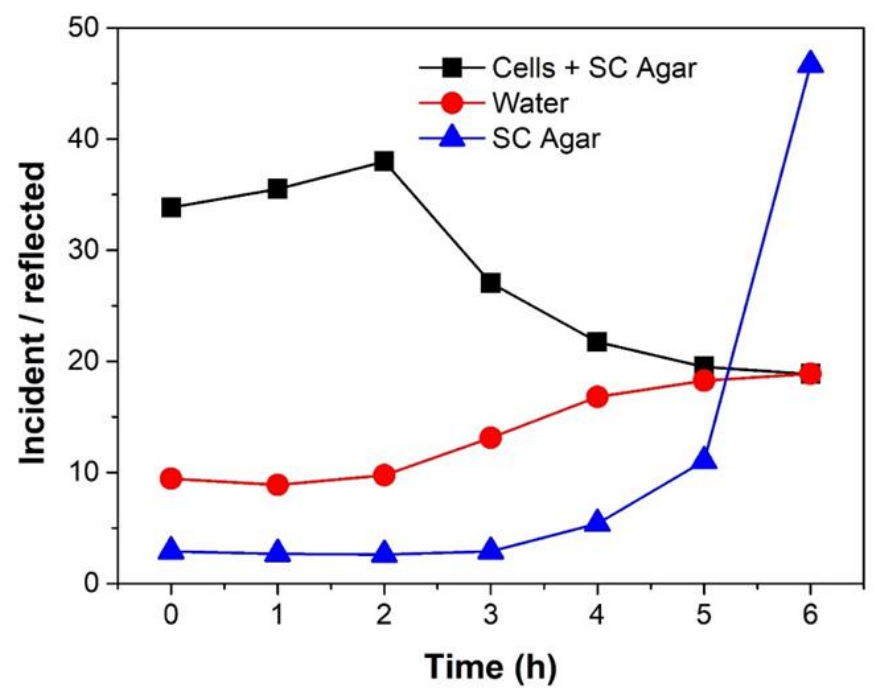

Fig. 5. Absorbed power as a ratio of incident and reflected power from yeast cells spotted on Synthetic Complete (SC) agar, blank water surface and blank Synthetic Complete (SC) agar surface respectively. Experiments were performed at a frequency of $85 \mathrm{GHz}$ with the amplitude of $5 \mathrm{dBm}$.

It can be seen from the figure that the sample absorbs a part of the incident power and the remaining is reflected. Hence, the ratio between the incident and reflected power values indicates the absorbed power of the sample under irradiation. We find that the yeast cells spotted on SC agar absorb more power as compared to plain water and blank SC agar. Interestingly, plain SC agar reflected most of the incident power. The experiment demonstrates that cells have a high absorbance of MMW irradiation because they are made mostly of water in a confined volume. 
In order to ascertain the relation between MMW absorption and size of sample irradiated as indicated from the previous experiments, different volumes of water were irradiated using horn antenna. Irradiation conditions of frequency, power and duration of exposure were kept constant as mentioned above to maintain stringency of analyses. Figure $6 \mathrm{a}$ shows the temperature rise measured using a digital thermometer during exposure involving a horn antenna on a large volume of water $(6500 \mu \mathrm{l})$. A $2^{\circ} \mathrm{C}$ rise of temperature is associated with the 6 hours duration of exposure. Figure $6 \mathrm{~b}$ shows the temperature rise involving a horn antenna on a smaller volume of water $(250 \mu \mathrm{l})$. The power density emitted by the antenna is given in Table 1. Reduction in the volume of the irradiated sample led to an increase of $1{ }^{\circ} \mathrm{C}$ in the rate of temperature rise (Fig. 6b). Figure $6 \mathrm{c}$ shows the rise of temperature during exposure involving a waveguide on the small volume of water $(250 \mu \mathrm{l})$ at $306.6 \mathrm{~mW} / \mathrm{cm}^{2}$. Contrary to the expectation of the waveguide causing thermal ablation due to higher power density, Figure $6 \mathrm{c}$ demonstrates that thermal effects were practically absent in our irradiation setup. Therefore, under conditions of constant frequency and power; the rise in temperature is inversely proportional to the volume of the sample irradiated. Finally, this experiment also confirms the hypothesis that biological cells exhibit high absorbance of MMW irradiation being constituted of water in a confined volume. The experiment demonstrates that MMW irradiation under the listed parameters raises temperatures up to $22{ }^{\circ} \mathrm{C}$ and does not cause thermal stress on yeast cells which grow at a physiological temperature of $30^{\circ} \mathrm{C}$.
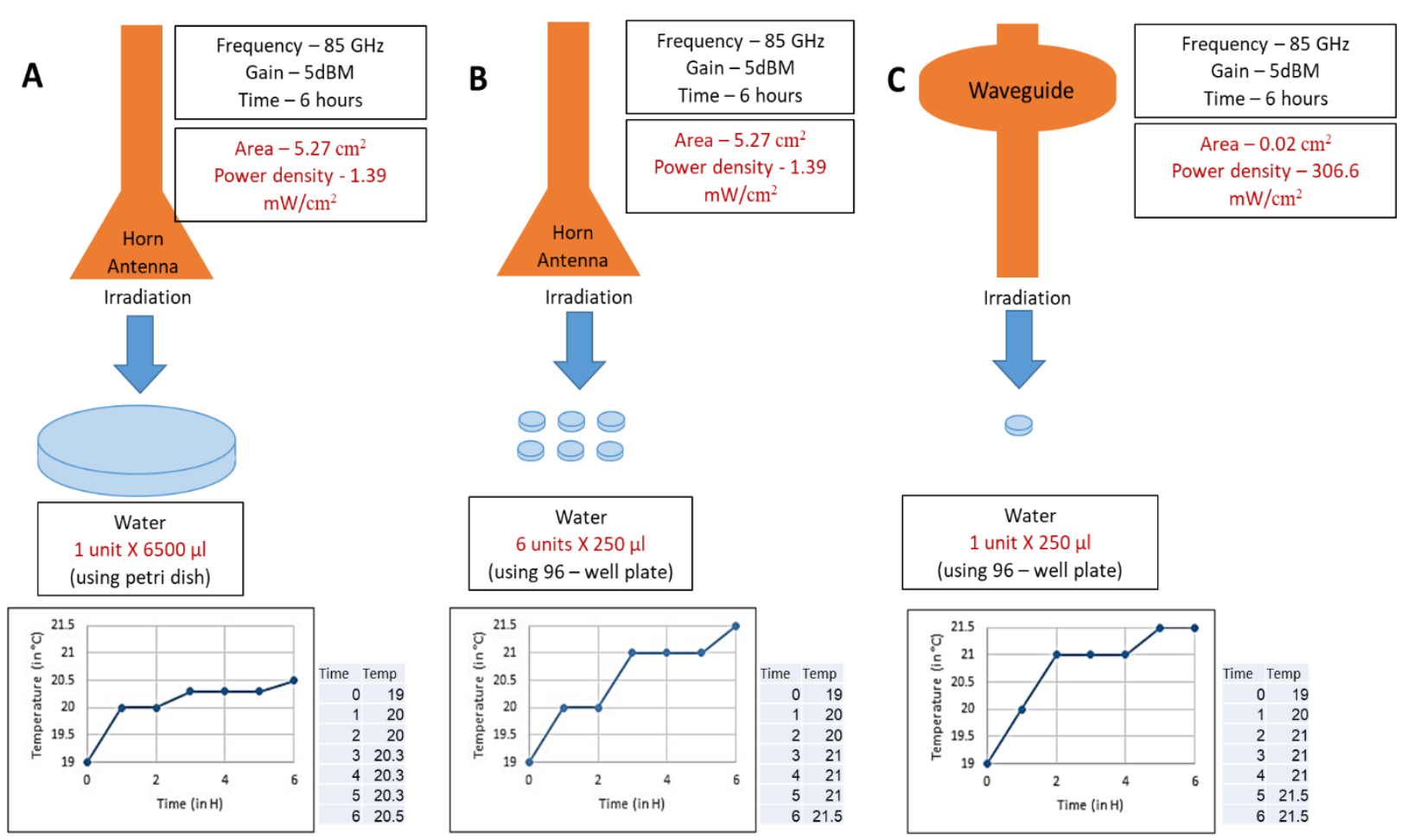

Fig. 6 Comparis on of temperature rise during MMW exposure involving horn antenna and wave guide. Indicated volumes of water were irradiated as illustrated. (a) Temperature rise of $6500 \mu \mathrm{l}$ water during MMW exposure at - $1.39 \mathrm{~mW} / \mathrm{cm}^{2}$. . (b) Temperature rise of $250 \mu \mathrm{l}$ water during MMW exposure at $-1.39 \mathrm{~mW} / \mathrm{cm}^{2}$. (c) Temperature rise of $250 \mu \mathrm{l}$ water during MMW exposure at $-306.6 \mathrm{~mW} / \mathrm{cm}^{2}$. Frequency, power and duration of exposure were kept constant at the values indicated.

A wave-guide provides a more focused beam of irradiation as compared to a horn antenna (Figires 1 and 6). We performed MMW irradiation (85 GHz, $5 \mathrm{dBm})$ of 
Saccharomyces cerevisiae BY4741 WT yeast cells using an open-ended waveguide (see methods). Single frequency at $85 \mathrm{GHz}$ is used because it affects WT yeast cell growth to the same extent as for all the other examined MMW frequencies (ref. Fig 3a). The thickness of agar medium and the number of cells were kept the same as those mentioned in the previous experiments. Cells were observed to be liquidated within 3 - 4 hours of treatment with 6.132 $\mathrm{mW}$ power and $306.6 \mathrm{~mW} / \mathrm{cm}^{2}$ power density. Further incubation of these cells under physiological conditions (at $30^{\circ} \mathrm{C}$ for 2 days) did not yield any colony growth. The experime nt demonstrates that MMW irradiation using an open-ended waveguide at a power density 300 times stronger than the one involving a horn antenna (ref. Fig. 3) completely terminates cell growth and division. Previously, we have reported human lung cancer cell specific-effect of MMW irradiation $[4,5,18]$. The treatment inhibited cancerous cell proliferation without affecting normal cell division of physiological tissue. The mechanism of this effect is partially explained in this study using the BY4741 Saccharomyces cerevisiae yeast strain as a model system. This mechanism of targeted cell growth inhibition allows it to be adapted for treatment of tumor metastasis. 


\section{Conclusions}

In this study, we explored the mechanism of MMW (85-105 GHz) irradiation on cell division using Saccharomyces Cerevisiae yeast cells as a model system. A standard horn antenna was used for MMW propagation, and the radiated power was measured in the far-field region using a similar antenna. The use of a horn antenna guarantees the frequency and power stabilities of the output signal. A comparative analysis of changes in the growth rate and cell viability of the control versus irradiated cells were performed. MMW irradiation decreases the growth rate of irradiated cells at a power density of about $1.0 \mathrm{~mW} / \mathrm{cm}^{2}$. Our results demonstrate that non-thermal MMW irradiation has the potential for future use in treating pathogenic fungal infections. Additionally, we study and report no mutagenic effects arising from this nonionizing radiation therapy. Our experiments demonstrate that the MMW irradiation allows the cells to retain their unmodified genetic material and likely affects the proteome by interacting with the water molecules. This accounts for the observed phenomenon of inhibited cell growth without genetic perturbation.

\section{Acknowledgment}

Shailendra Rajput is thankful to the Israeli Council for Higher Education (CHE) for fellowship. This work was supported in part by the Ariel Center for Applied Cancer Research.

\section{Conflicts of Interest}

The authors declare no conflict of interest.

\section{References}

1. Koo MM, Swann R, McPhail S, Abel, GA, Elliss-Brookes, L Rubin GP, Lyratzopoulos, G. Presenting symptoms of cancer and stage at diagnosis: evidence from a cross-sectional, population-based study. 2020. Lancet Oncology, Vol. 21, Issue 1, P73-79, doi: 10.1016/S1470-2045(19)30595-9

2. Ryan JL. 2012. Ionizing radiation: the good, the bad, and the ugly. J Invest Dermatol 132:985-993.

3. Salvo N, Barnes E, Van Draanen J, Stacey E, Mitera G, Breen D, De Angelis C. 2010. Prophylaxis and management of acute radiation-induced skin reactions: a systematic review of the literature. Curr Oncol 17:94.

4. Komoshvili K, Becker T, Levitan J, Yahalom A, Barbora A., Liberman-Aronov S Morphological Changes in H1299 Human Lung Cancer Cells Following MillimeterWave Irradiation. Applied Sciences, Volume $10 \quad$ (9) 3187; https//doi.org/10.3390/app10093187

5. Komoshvili K, Israel K, Levitan J, Yahalom A, Barbora A., Liberman-Aronov S. "WBand Millimeter Waves Targeted Mortality of H1299 Human Lung Cancer Cells without Affecting Non-Tumorigenic MCF-10A Human Epithelial Cells In Vitro." Applied Sciences 10 (14) 4813. https://doi.org/10.3390/app10144813

6. A. Mirbeik-Sabzevari and N. Tavassolian, "Ultrawideband, Stable Normal and Cancer Skin Tissue Phantoms for Millimeter-Wave Skin Cancer Imaging," in IEEE Transactions on Biomedical Engineering, vol. 66, no. 1, pp. 176-186, Jan. 2019, doi: 10.1109/TBME.2018.2828311. 
7. Yu G, Coln EA, Schoenbach KH, Gellerman M, Fox P, Rec L, Liu, S. 2002. A study on biological effects of low-intensity millimeter waves. IEEE T Plasma Sci 30:14891496.

8. Ziskin, MC. 2006. Physiological mechanisms underlying millimeter wave therapy. In Bioelectromagnetics Current Concepts, Springer, pp 241-251.

9. Altmann K, Dürr M, Westermann B. 2007. Saccharomyces cerevisiae as a model organism to study mitochondrial biology. In Mitochondria, Springer, pp 81-90.

10. Beck H, Dobritzsch D, Piškur J. 2008. Saccharomyces kluyveri as a model organism to study pyrimidine degradation. FEMS Yeast Res 8:1209-1213.

11. Kachroo AH, Laurent JM, Yellman CM, Meyer AG, Wilke CO, Marcotte EM. 2015.

Systematic humanization of yeast genes reveals conserved functions and genetic modularity. Science 348:921-925.

12. Grundler W. 1992. Intensity-and frequency-dependent effects of microwaves on cell growth rates. J Electroanal Chem 342:361-365.

13. Grundler W, Keilmann F, Fröhlich H. 1977. Resonant growth rate response of yeast cells irradiated by weak microwaves. Phys Lett A 62:463-466.

14. Furia L, Hill DW, Gandhi OP. 1986. Effect of millimeter-wave irradiation on growth of Saccharomyces cerevisiae. IEEE T Bio-Med Eng 11:993-999.

15. Vojisavljevic V, Alsuhaim H, Pirogova E. 2016. Low power microwave exposures at $968 \mathrm{MHz}$ increase the growth rate of Breanomyces bruxellensis yeast cells. Paper presented at the 2016 IEEE International Conference on Microwave and Millimeter Wave Technology.

16. Smolyanskaya A, Vilenskaya R. 1974. Effects of millimeter-band electromagnetic radiation on the functional activity of certain genetic elements of bacterial cells. Sov Phys USPEKHI 16:571.

17. Rajput S, Komoshvili K, Aronov S, Barbora A, Patnaik P, Levitan J, Yahalom A. 2019. Optimization of transmitted power of horn antenna for biomedical applications. Paper presented at the 2019 IEEE International Conference on Microwaves, Antennas, Communications and Electronic Systems.

18. Komoshvili K, Levitan J, Aronov S, Kapilevich B, Yahalom A. 2011. Millimeter waves non-thermal effect on human lung cancer cells. Paper presented at the $2011 \mathrm{IEEE}$ International Conference on Microwaves, Communications, Antennas and Electronic Systems.

19. Game J. 1983. Radiation-sensitive mutants and repair in yeast. In Yeast genetics, Springer, pp 109-137.

20. Game J. 1993. DNA double-strand breaks and the RAD50-RAD57 genes in Saccharomyces. Semin Cancer Bio 4:73-83.

21. Edwards G, Davis C, Saffer J, Swicord M. 1984. Resonant microwave absorption of selected DNA molecules. Phys Rev Lett 53:1284.

22. Belyaev IY, Alipov YD, Polunin VA, Shcheglov VS. 1993. Evidence for dependence of resonant frequency of millimeter wave interaction with Escherichia coli K12 cells on haploid genome length. Electro-and Magnetobiology 12:39-49.

23. Koyama S, Narita E, Shimizu Y, Suzuki Y, Shïna T, Taki M, Miyakoshi J. 2016. Effects of long-term exposure to $60 \mathrm{GHz}$ millimeter-wavelength radiation on the genotoxicity and heat shock protein (HSP) expression of cells derived from human eye. Int J Environ Res Public Health, 13:802. 
24. Lunkenheimer P, Emmert S, Gulich R, Köhler M, Wolf M, Schwab M, Loidl A. 2017. Electromagnetic-radiation absorption by water. Phys Rev E 96:062607.

25. Rogacheva SM, Babaeva MI. 2013. The effect of millimeter waves at the yeast Saccharomyces cerevisiae during heliogeophysical disturbances. Paper presented at the Saratov Fall Meeting 2012: Optical Technologies in Biophysics and Medicine XIV; and Laser Physics and Photonics XIV.

26. White J. 1952. Variation in water content of yeast cells caused by varying temperatures of growth and by other cultural conditions. J Inst Brewing 58:47-50.

27. Bellissent-Funel MC, Hassanali A, Havenith M, Henchman R, Pohl P, Sterpone F, Garcia AE. 2016. Water determines the structure and dynamics of proteins. Chem Rev 116:7673-7697.

28. O'Brien EP, Brooks BR, Thirumalai D. 2012. Effects of $\mathrm{pH}$ on proteins: predictions for ensemble and single-molecule pulling experiments. J Am Chem Soc 134:979-987.

29. Di Russo NV, Estrin DA, Martí MA, Roitberg AE. 2012.pH-dependent conformational changes in proteins and their effect on experimental pKas: The case of nitrophorin 4. PLoS Comput Bio 8:e1002761. 